Camila Soares (Orcid: 0000-0002-2076-0758) ${ }^{1}$ Anderson Alves Dias (Orcid: 0000-0002-9154-3849) ${ }^{1}$ Thaís de Souza Toledo (Orcid: 0000-0002-8530-5764) Jéssica Carvalho Lima (Orcid: 0000-0003-0972-1886) Patricia Ribeiro Marcacine (Orcid: 0000-0003-1784-2231) ${ }^{2}$ Dernival Bertoncello (Orcid: 0000-0002-4432-4651) (1,3 $^{3}$ Isabel Aparecida Porcatti de Walsh (Orcid: 0000-0002-2317-1326) (1,3 $^{1,3}$

\section{LESÕES POR ESFORÇOS REPETITIVOS E DISTÚRBIOS OSTEOMUSCULARES RELACIONADOS AO TRABALHO (LER/DORT): PAPEL DOS PROFISSIONAIS DA SAÚDE}

\author{
REPETITIVE STRAIN INJURIES AND WORK-RELATED \\ MUSCULOSKELETAL DISORDERS (RSI / WMSD): ROLE OF \\ HEALTH PROFESSIONALS
}

Autor correspondente:

Isabel Aparecida Porcatti de Walsh

E-mail: isabelpwalsh@gmail.com

\section{RESUMO}

Entre as causas de absenteísmo e presenteísmo no trabalho, estão as Lesões por Esforços Repetitivos e os Distúrbios Osteomusculares Relacionados ao Trabalho (LER/DORT), gerando perda de produtividade e ocasionando importante impacto econômico para o empregador, governo, sociedade, além de limitações e sofrimento aos trabalhadores acometidos, evidenciando a necessidade de assistência específica e qualificada para esses distúrbios e estratégias de prevenção perante eles. A responsabilidade dos profissionais de saúde vai além do diagnóstico e do tratamento adequado, envolvendo as condutas a serem tomadas não só na área clínica, mas também nas áreas previdenciária, trabalhista, de responsabilidade civil e, às vezes, até criminal. Nesse sentido, os órgãos representantes das categorias e as Instituições de Ensino Superior têm papel importante para divulgar, explicitar melhor a área e trabalhar mais as disciplinas relacionadas com a Saúde do Trabalhador, bem como conduzir esses profissionais ao que lhes cabe realizar nessa área e, principalmente, refletir sobre sua responsabilidade profissional, política e social com relação às questões referentes à precarização do trabalho e à exclusão social, com um olhar para a integralidade da atenção. Além disso, é essencial que os profissionais da saúde cumpram com sua responsabilidade na notificação desses agravos, para que seja conhecida a realidade da população trabalhadora, gerando subsídios para a tomada de decisões dos órgãos competentes dos governos.

PALAVRAS-CHAVE: Saúde do Trabalhador; Distúrbios Osteomusculares Relacionados ao Trabalho; Profissionais de saúde.

\begin{abstract}
Among the causes of absenteeism and presenteeism at work are Repetitive Strain Injuries and Work-Related Musculoskeletal Disorders (RSI / WMSD), generating loss of productivity and causing an important economic impact for the employer, government, society, in addition to limitations and suffering for the affected workers, showing the need for specific and qualified assistance for these disorders and prevention strategies before them. The responsibility of health professionals goes beyond diagnosis and proper treatment, involving the conducts to be taken not only in the clinical area, but also in the social security, labor, civil liability and, sometimes, even criminal areas. In this sense, the organs representing the categories and Higher Education Institutions have an important role in disseminating, explaining the area better and working more on the subjects related to Occupational Health, as well as guiding these professionals to what they have to do in this area and, mainly, reflect on their professional, political and social responsibility in relation to issues related to job insecurity and social exclusion, with a view to comprehensive care. In addition, it is essential that health professionals comply with their responsibility in the notification of these diseases, so that the reality of the working population is known, generating subsidies for decision-making by the competent government agencies.
\end{abstract}




\section{ADOECIMENTO E TRABALHO}

Investigações que analisam dimensões do trabalho e emprego em alguns estados brasileiros indicam que a relação entre a proporção de trabalhadores que têm jornada laboral acima da jornada legal semanal de 44 horas semanais ultrapassa 37,8\% ${ }^{1}$. Ainda, observa-se, com relação ao tempo médio de procura por uma nova ocupação, que $38,8 \%$ estavam há, pelo menos, um ano nessa situação, no terceiro trimestre de $2019^{2}$. Tal fato sugere que o medo de perder o emprego obriga o trabalhador a submeter-se a condições de trabalho que ultrapassam sua capacidade física e mental, impactando na sua saúde geral ${ }^{3}$.

Nesse sentido, o presenteísmo se dá quando o trabalhador vai ao trabalho, apesar de sua condição de saúde fragilizada, implicando o ato ou comportamento de comparecer, ou seja, de estar fisicamente presente no local de trabalho, mas, devido a problemas de doença ou de outra condição médica, ser incapaz de produzir o que, em outras circunstâncias, considerou-se adequado. Entre as possibilidades de motivação que isso aconteça, estão: o trabalhador não se julga suficientemente doente para interromper suas atividades profissionais e dedicar-se ao seu cuidado; tem pendências financeiras que poderiam se agravar se, em virtude de seu afastamento, perder o emprego ou tiver descontadas de seu salário o tempo não trabalhado ou a meta de produtividade não alcançada; medo de perder o emprego; medo de ser substituído definitivamente por seu substituto temporário; medo do julgamento alheio sobre sua situação de fragilidade; medo de não ser aceito; medo de sobrecarregar os pares; medo de ser penalizado de alguma forma e medo de perder oportunidades de crescimento na carreira $^{4-6}$

Já o absenteísmo (período de ausência laboral atribuída a uma incapacidade do indivíduo, contabilizado o período de duração da licença médica devido à incapacidade ao trabalho por doença, acidente ou lesão) se caracteriza por ser um fenômeno multidimensional e complexo no campo da Saúde do Trabalhador, pois envolve a interação de diversos fatores laborais ligados às condições de trabalho que podem ocasionar agravos à saúde, decorrentes da natureza ou do conteúdo da atividade exercida, da presença de fatores psicossociais ou, até mesmo, de fatores econômicos e sociais indissociáveis do trabalho e que influenciam, diretamente, na saúde e na segurança dos trabalhadores ${ }^{7,8}$.

\section{LESÕES POR ESFORÇOS REPETITIVOS/DIS- TÚRBIOS OSTEOMUSCULARES RELACIONA- DOS AO TRABALHO (LER/DORT)}

Entre as causas de absenteísmo e presenteísmo no trabalho, estão as afecções osteomusculares, gerando perda de produtividade e ocasionando importante impacto econômico para o empregador, governo e sociedade, além de limitações e sofrimento aos trabalhadores acometidos, ficando assim evidenciada a necessidade de assistência específica e qualificada para esses distúrbios, possibilitando novas estratégias de prevenção diante desses agravos.

Essas afecções, conhecidas no Brasil como Lesões por Esforços Repetitivos/Distúrbios Osteomusculares Relacionados ao Trabalho (LER/DORT), manifestam-se por meio de sinais e sintomas, como dor, parestesia, sensação de peso e fadiga, principalmente nos membros superiores, que aparecem vagarosamente, podendo ser concomitantes ou não, e que causam, frequentemente, incapacidade laboral temporária ou permanente ${ }^{9,10}$.

Sua etiologia é multifatorial e complexa, envolvendo aspectos biomecânicos, cognitivos, sensoriais, afetivos e psicossociais e fatores relacionados com as condições e à organização do trabalho ${ }^{11}$, podendo ter relação com a postura incorreta ou mantida exigida pelas tarefas; fatores ambientais, como a temperatura extrema; ruído; dimensões do posto de trabalho; vibrações; pressões mecânicas; logística de trabalho incorreta; coação para aumento da produtividade; invariabilidade da tarefa. A organização do trabalho é apontada como uma das 
principais responsáveis pelo grande número de trabalhadores com LER/DORT, que afirmam que estas são resultados da superutilização do sistema osteomuscular, instalando-se progressivamente no trabalhador sujeito a fatores de risco técnico-organizacionais ${ }^{12}$.

Estão entre as doenças que mais afetam os trabalhadores brasileiros. Utilizando dados do Sistema de Informação de Agravos de Notificação (Sinan), o levantamento aponta que, entre os anos de 2007 e 2016, 67.599 casos de LER/DORT foram notificados à pasta. Nesse período, o total de registros cresceu $184 \%$, passando de 3.212 casos, em 2007, para 9.122, em 2016. Tanto o volume quanto o aumento nos casos nesse período sinalizam alerta em relação à saúde dos trabalhadores ${ }^{13}$. Ainda, a pesquisa nacional de saúde, em 2013, identificou que, no Brasil, 2,4\% dos trabalhadores (3.568.095 pessoas com mais de 18 anos) entrevistados declararam ter diagnóstico médico de LER/DORT ${ }^{14,15}$, dos quais, mais da metade afirmou que a doença limitava suas atividades habituais, sendo que, para $13,1 \%$, essa limitação era intensa e, para $2,8 \%$, muito intensa ${ }^{15}$.

Muitas vezes, as LER/DORT iniciam-se com quadro clínico de dor que não é possível mensurar objetivamente e que retarda o diagnóstico. Associado ao medo do desemprego, o trabalhador tende a se desligar dos sofrimentos físico e psíquico, conduzindo-se ao individualismo e à submissão aos riscos do trabalho ${ }^{16} \mathrm{e}$ à dor inicial, que, na maioria das vezes, é "tratada" com automedicação ${ }^{17,18}$. Dessa forma, o silêncio coletivo em relação à doença e ao sofrimento torna-se uma estratégia de defesa que protege o trabalhador da angústia do medo das consequências dela ${ }^{19}$.

Quando a dor começa a repercutir nas atividades da vida diária e laborais, os sujeitos passam a praticar a automedicação para alívio de sintomas dolorosos e para o atendimento das demandas de trabalho. Diante do agravamento dos sintomas, as unidades de pronto atendimento começam a ser frequentadas pelo trabalhador com dor e limitação laboral. No entanto, nos atendimentos, em geral, não há nenhuma recomendação para afastamento prolongado das atividades de trabalho nem restrições de atividades laborais relacionadas com os esforços físicos de risco. O objetivo concentra-se em torno de medicar a dor sem aprofundar nas possíveis causas relacionadas com o trabalho ${ }^{17}$.

Os trabalhadores que apresentam LER/ DORT possuem um quadro clínico complexo. Dessa maneira, é necessário que o profissional de saúde faça uma investigação adequada. Nesse sentido, a Instrução Normativa no 98 , de 5 de dezembro de 2003, que aprova Norma Técnica sobre LER/DORT, indica que a conclusão diagnóstica deve considerar o quadro clínico, sua evolução, fatores etiológicos possíveis, com destaque para a anamnese e fatores ocupacionais $^{20}$.

No entanto, desafio maior é o cumprimento das normativas que orientam a prática clínica quanto à avaliação para o estabelecimento do nexo causal com o trabalho, para orientar/encaminhar o trabalhador, com vistas aos seus direitos trabalhistas e previdenciários assegurados.

\section{O PAPEL DOS PROFISSIONAIS DE SAÚDE NO DIAGNÓSTICO E TRATAMENTO DAS LER/ DORT}

Seja no pronto atendimento, seja na Atenção Básica ou secundária (especializada), o que se observa é que os profissionais de saúde possuem dificuldade em entender a complexidade que envolve a LER/DORT e o seu nexo causal, que engloba a doença e o trabalho. A atribuição de estabelecer nexo de causa e/ou agravamento entre as condições de trabalho e o quadro clínico não é exclusividade de nenhum profissional em especial. Essa etapa é multidisciplinar, pois pressupõe o conhecimento da técnica de extrair informações do trabalhador por meio de um aprofundamento da anamnese ocupacional ${ }^{11}$.

Além do diagnóstico adequado desses distúrbios, caracterizando sua relação com o trabalho 
e, assim, reconhecendo-os como das LER/DORT, é necessário que os acometidos recebam o tratamento adequado. Este requer uma abordagem interdisciplinar, por meio da atuação integrada de profissionais de diferentes áreas, cujo objetivo principal seja explorar as potencialidades remanescentes, a melhora da qualidade de vida e da capacidade funcional, e não apenas o alívio da dor, como ocorre nos modelos tradicionais de reabilitação de trabalhadores ${ }^{21,22}$, de modo que seja possível sua reinserção em um ambiente laboral adequado e na sociedade.

A atenção pode acontecer de forma individual ou em grupo, sendo que as terapias coletivas resultam em grandes ganhos para os trabalhadores acometidos, uma vez que terão uma visão coletiva do seu problema e vivências que favorecerão o autocuidado e a autonomia diante de seu quadro clínico e limitações impostas pela doença, buscando adaptações em sua forma de trabalhar e de realizar as atividades de vida diária, suavizando o sofrimento advindo da doença e a ideologia de culpabilização desses trabalhadores, permitindo a transformação das percepções individuais em coletivas, além de ser um espaço de discussão e partilha de conhecimento, direitos e outros temas, auxiliando no estabelecimento de relações do seu próprio adoecimento com o processo de trabalho, de forma a fomentar sua consciência crítica e adoção de ações transformadoras dos ambientes de trabalho e da realidade social, representando um espaço de socialização e de reconstrução de sua cidadania, que motiva a superação da problemática e favorece o retorno ao trabalho ${ }^{23,24}$.

No entanto, a responsabilidade desses profissionais diante desses distúrbios vão para além do diagnóstico e tratamento adequado, no enfrentamento de desafios que se situam na essência das relações sociais de produção, em que sobressaem os conflitos de classe e a hegemonia do poder político-econômico na condução das políticas públicas $\mathrm{O}$ diagnóstico das LER/DORT envolve também as condutas que devem ser tomadas nas áreas previdenciária, trabalhista, de responsabilidade civil e, às vezes, até criminal $^{12}$.
As empresas têm um certo controle privado da saúde e segurança dos trabalhadores, a partir de Serviços Especializados em Engenharia de Segurança e Medicina do Trabalho (SESMT). Entretanto, com a participação mínima do Estado, essas equipes estão subordinadas à vontade do empregador, bem como assumem papel, na maioria das vezes, de apenas medicalizar a dor desqualificando as ações multidisciplinares e o sofrimento a favor da produção ${ }^{19}$.

O modelo neoliberal, associado à pouca intervenção do Estado no mercado, impõe grande desafio nesse campo, para que haja uma ação conjunta e integrada do Estado, tornando mais incisiva a atenção às políticas públicas, tendo em vista a responsabilização das empresas pela produção do adoecimento no trabalho, levando em consideração a saúde e as empresas, com a finalidade de ocorrer melhorias nos processos de prevenção das LER/ DORT, bem como o tratamento e reabilitação dos trabalhadores acometidos ${ }^{18}$.

Desse modo, há muito o que fazer para consolidar a atuação dos profissionais da saúde nessa área. Nesse sentido, os órgãos representantes da categoria e as Instituições de Ensino Superior têm papel importante para divulgar, explicitar e trabalhar mais as disciplinas relacionadas com a Saúde do Trabalhador, bem como conduzir esses profissionais ao que lhes cabe realizar nessa área e, principalmente, refletir sobre sua responsabilidade profissional, política e social com relação às questões referentes à precarização do trabalho e à exclusão social, com um olhar para a integralidade da atenção ${ }^{25}$.

Enquanto participante do processo de consolidação de ações de vigilância, tem-se o desafio de compreender a abrangência de suas habilidades, o conhecimento do perfil epidemiológico de adoecimento dos trabalhadores e agregar em sua atuação novos elementos, possibilitando melhores condições para o desenvolvimento de ações de saúde integrais ${ }^{26}$. 
Finalmente, ao estabelecer o nexo causal entre a doença e o trabalho no caso das LER/DORT, faz-se necessário o registro oficial da doença, o que nem sempre é obtido ${ }^{27}$. Vale ressaltar que quando estas são reconhecidas, a empresa deve emitir a Comunicação de Acidente de Trabalho (CAT) ao Instituto Nacional do Seguro Social (INSS); e, que quando isso não ocorre, o próprio acidentado, seus dependentes, o sindicato, o médico que o assistiu ou qualquer autoridade pública poderão realizá-lo, visando garantir o acesso ao benefício auxílio-doença acidentário, garantindo 12 meses de estabilidade na empresa ao trabalhador quando retornar ao trabalho, entre outros ${ }^{28}$. A CAT é uma importante fonte de informações epidemiológicas, embora se refira apenas aos trabalhadores cobertos pelo Seguro Acidente de Trabalho, sendo, portanto excluídos dessa fonte de dados os trabalhadores autônomos, domésticos, funcionários públicos estatutários, subempregados, muitos trabalhadores rurais, entre outros ${ }^{27}$.

No entanto, considerando os dados do levantamento feito a partir dos microdados da Pesquisa Nacional por Amostra de Domicílios Contínua (PNAD Contínua), de que 62,4\% das pessoas que trabalham na informalidade estão no interior, contemplando 20,8 milhões de trabalhadores sem carteira assinada (empregados do setor privado e trabalhadores domésticos), sem CNPJ e sem contribuição para a previdência oficial (empregadores e por conta própria) ou sem remuneração (auxiliam em trabalhos para a família), sendo que $36,3 \%$ da população ocupada está em uma dessas condições de informalidade $^{29}$, fazem-se necessários outros instrumentos para que os números estatísticos relacionados com as LER/DORT correspondam à realidade de toda a população trabalhadora.

Nesse sentido, desde 2004, o Ministério da Saúde tornou obrigatória a notificação de 11 agravos e doenças relacionadas com o trabalho, entre eles, as LER/DORT, atualmente em vigor por meio da PORTARIA DE CONSOLIDAÇÃO No 4 , DE 28 DE SETEMBRO DE $2017^{30}$. A notificação compulsória é obrigatória a todos os profissionais de saúde no exercício da profissão, bem como os responsáveis por organizações e estabelecimentos públicos e particulares de saúde, devendo ser feita para qualquer trabalhador, independentemente de seu vínculo de trabalho.

Assim, é essencial que os profissionais da saúde cumpram também com sua responsabilidade na notificação desses agravos, para que seja conhecida a realidade da população trabalhadora, gerando subsídios para a tomada de decisões dos órgãos competentes dos governos.

\section{REFERÊNCIAS}

1. Cardoso MCA. Organização e Intensificação do Tempo de Trabalho. Sociedade e Estado, Brasília, DF, v. 28, p. 351-374; 2013.

\section{Lameiras MAP, Corseuil CHL, Ramos LRA, Car-} valho SS. Seção VIII Mercado de trabalho Mercado de trabalho. Carta de Conjuntura $|45| 4^{\circ}$ trimestre de 2019 [acessado 2020 fev 5]. Disponível em: http://www.ipea.gov.br/portal/images/ stories/PDFs/conjuntura/191212_cc_45_mercado_de_trabalho.pdf.

3. Cardoso ACM, Ribeiro D, Morgado LP, Linhares R. A saúde do trabalhador no processo de negociação coletiva no Brasil. Estudos e pesquisas, n. 76. São Paulo: Dieese; 2015.

4. Altoé A. Políticas Institucionais e seus desdobramentos sobre o trabalho docente: absenteísmo e presenteísmo [dissertação]. Belo Horizonte (MG): Pontifícia Universidade Católica de Minas Gerais - PUCMG; 2010.

5. Hemp P. Presenteeism: at work - but out of it. Harv Bus Rev 2004; 82(10):49-58 
6. Silva BMCC. Prevalência do presenteísmo em trabalhadores de uma indústria do setor alimentício [dissertação]. Campinas (SP): Universidade Estadual de Campinas - Unicamp, Faculdade de Ciências Médicas; 2015.

7. Isah EC, Omorogbe VE, Oyovwe L. Self reported absenteeism among hospital workers in benin city, Nigeria. Ghana Med J 2008 ;42(1):2-7.

8. Mininel VA, Felli VEA, Silva EJ, Torri Z, Abreu NA, Branco MTAF. Cargas de trabalho, processos de desgaste e absenteísmo-doença em enfermagem. Rev Latino-Am Enfermagem 2013; 21(6):1290-7.

9. Brasil. Ministério da Saúde. Secretaria de Vigilância em Saúde. Departamento de Vigilância em Saúde Ambiental e Saúde do Trabalhador. Dor relacionada ao trabalho: lesões por esforços repetitivos (LER): distúrbios osteomusculares relacionados ao trabalho (Dort) / Ministério da Saúde, Secretaria de Vigilância em Saúde, Departamento de Vigilância em Saúde Ambiental e Saúde do Trabalhador. - Brasília: Editora do Ministério da Saúde; 2012

10. Brasil. Ministério da Saúde. Protocolo de investigação, diagnóstico, tratamento e prevenção de Lesão por Esforços Repetitivos/Distúrbios Osteomusculares Relacionados ao Trabalho. [internet]. Diário Oficial da União 2000 [acessado 2019 jun 20]; 19 jul. Disponível em: http://bvsms.saude.gov.br/bvs/publicacoes/ protocolo_ler.pdf

11. Brasil. Ministério da Saúde. Secretaria de Vigilância em Saúde. Departamento de Vigilância em Saúde Ambiental e Saúde do Trabalhador. Dor relacionada ao trabalho: lesões por esforços repetitivos (LER): distúrbios osteomusculares relacionados ao trabalho (Dort). [internet]. Brasília: Ministério da Saúde; 2012 [acessado 2019 jun 22]. 68 p. (Série A. Normas e Manuais Técnicos); (Saúde do Trabalhador;
10. Protocolos de Complexidade Diferenciada). Disponível em: http://bvsms.saude.gov. br/bvs/publicacoes/dor_relacionada_trabalho_ler_dort.pdf.

12. Brasil. Ministério da Saúde. Diagnóstico, tratamento, reabilitação, prevenção e fisiopatologia das LER/DORT. [internet]. Série A: Normas e manuais técnicos n. 105, 64 p. Brasília, DF: 2001 [acessado 2019 jun 6]. Disponível em: http://bvsms.saude.gov.br/bvs/publicacoes/ diag_tratamento_ler_dort.pdf

13. Brasil. Ministério da Saúde. LER e DORT são as doenças que mais acometem os trabalhadores, aponta estudo. [internet]. 2019 [acessado 2019 jun 6]. Disponível em: https:/www.saude.gov.br/noticias/agencia-saude/45404-ler-e-dort-sao-as-doencas-que-mais-acometem-os-trabalhadores-aponta-estudo.

14. Assunção AÁ, Abreu MNS. Factor associated with self-reported work-related musculoskeletal disorders in Brazilian adults. Rev Saúde Pública 2017; 51(suppl 10):1-10s.

15. Maeno M, Tavares DS, Lima CQB. Dia Internacional de Combate às LER/Dort. Fundacentro; 2017.

16. Merlo ÁRC, Lapis NL. A saúde e os processos de trabalho no capitalismo: reflexões na interface da psicodinâmica do trabalho e da sociologia do trabalho. Psicol Soc 2007; 19(1):61-8.

17. Zavarizzi CP, Alencar MCB. Afastamento do trabalho e os percursos terapêuticos de trabalhadores acometidos por LER/Dort. Saúde Debate [internet] 2018 [acessado 2019 jun 6]; 42(116):113-24. Disponível em: http://www. scielo.br/scielo.php?script=sci_abstr

18. Dal Magro MLP, Coutinho MC. O obscurecimento da dor como dispositivo de controle da força de trabalho frente às LER/Dort: o caso das indústrias de abate e processamento de carnes. Univ Psychol 2013; 12(4):1197-211. 
19. Dejours C. A Loucura do Trabalho. 6th ed. São Paulo: Cortez; 2015. 222 p.

20. Brasil. Ministério da Previdência Social. Instrução Normativa 99/2003. Aprova Norma Técnica sobre Lesões por Esforços Repetitivos-LER ou Distúrbios Osteomusculares Relacionados ao Trabalho DORT; 2003.

21. Maeno M, organizador. Dor relacionada ao trabalho: Lesões por Esforços Repetitivos (LER): Distúrbios Osteomusculares Relacionados ao Trabalho (DORT): Protocolos de atenção integral à Saúde do Trabalhador de Complexidade Diferenciada [Internet]. Brasília: Ministério da Saúde; 2012 [acessado 2017 jun 2]. Disponível em: http://bvsms. saude.gov.br/bvs/publicacoes/dor_relacionada_trabalho_ler_dort.pdf.

22. Lima CQB, Tavares DS, Maeno M, organizadores. Proposta de diretrizes para uma política de reabilitação profissional. São Paulo: Fundacentro; 2013.

23. Merlo ARC, Jacques MGC, Hoefel MGL. Trabalho de grupo com portadores de LER/ DORT: relato de experiência. Psicol Reflex Crit 2001; 14(1):253-8.

24. Hoefel MG, Jacques MG, Amazarray MR, Mendes JMR, Netz JA. Uma proposta em Saúde do Trabalhador com portadores de LER/DORT: grupos de ação solidária. Cad Psicol Soc Trab 2004; 7:31-9.

25. Walsh IAP, Lima JC, Betoncello D. Fisioterapia e saúde do trabalhador no Brasil. Cad edu saúde e fis 2018; 5(9).
26. Melo BF, Souza ACAG, Ferrite S, Bernardes KO. Atuação do fisioterapeuta nos Centros de Referência em Saúde do Trabalhador: indicadores das notificações dos Dort. Fisioter Pesqui 2017; 24(2):136-142.

27. Leite PC, Silva A, Merighi MAB. A mulher trabalhadora de enfermagem e os distúrbios osteomusculares relacionados ao trabalho. Rev Esc Enferm. USP 2007 Jun; 41(2):287-91

28. Brasil. Ministério do Trabalho e Previdência Social. Comunicação de Acidente de Trabalho (CAT) [internet]. 2016 [acessado 2020 jan 30]. Disponível em: http://www.mtps.gov.br/servicos-do-ministerio/servicos-da-previdencia/ mais-procurados/aposentadoria-por-tempo-de-contribuicao/documentos-para-comprovacao-de-tempo-de-contribuicao/ comunicacao-de-acidente-de-trabalho-cat.

29. Brasil. Instituto Brasileiro de Geografia e Estatística. Pesquisa revela retrato inédito do mercado de trabalho do interior do país. [internet]. 2019 [acessado 2020 jan 30]. Disponível em: https://agenciadenoticias.ibge. gov.br/agencia-noticias/2012-agencia-de-noticias/noticias/25066-pesquisa-revela-retrato-inedito-do-mercado-de-trabalho-do-interior-do-pais

30. Brasil. Ministério da Saúde. Portaria de Consolidação No 4, de 28 de setembro de 2017. Disponível em: http://bvsms. saude.gov.br/bvs/saudelegis/gm/2017/ prc0004_03_10_2017.html. Acesso em: 04 set 2020 